EDITORIAL

\title{
Improving evidence based cardiac care and policy implementation over the patient journey: the potential of coronary heart disease registers
}

\author{
A M Clark, I N Findlay, for the Have a Heart Paisley IT Group
}

Heart 2005;91:1127-1130. doi: 10.1136/hrt.2004.051979

Coronary heart disease registers offer considerable potential for providing increased support for practitioners, facilitating improvements in patient care, and allowing efficient monitoring of care provision and outcomes

M uch hope was placed upon evidence based medicine to improve health care quality and outcomes. ${ }^{1}$ Despite the relative strength of the evidence base guiding cardiac care, many current practices for the treatment and secondary prevention of coronary heart disease (CHD) in Europe are suboptimal ${ }^{2}{ }^{3}$ and substantial inequalities in treatment and access remain common. ${ }^{4-6}$

Numerous health policies and guidelines that contain prescriptive targets or specific protocols for service providers have been introduced to facilitate evidence based cardiac care. ${ }^{7-12}$ As guidelines become increasingly specific and new evidence continues to accrue on an almost weekly basis, remaining cognisant of latest developments and implementing appropriate changes to practice is challenging for time pressed specialists, but is especially difficult for those in general practice or non-specialists. ${ }^{13} 14$ Though responsibility for suboptimal practice is often attributed to the individual clinician, a myriad of both individual and structural factors contribute to the lack of knowledge utilisation, including perceived costs, side effects, time management challenges, and a lack of local opinion leaders. ${ }^{15}$

\section{SYSTEMS BASED APPROACH}

In contrast to an individualistic approach, a systems based approach views the establishment of responsive and supportive structures as being vital to building care capacity. Rather than seeing the solution as providing more guidelines, this approach suggests that practitioners need better systems to inform their decision making. Yet, effective information systems are seldom in place to support the provision of rapid, equitable, and evidence based care. $^{13}{ }^{16}$ In this regard, the effective utilisation of information technology is central to the provision of modern cardiac care. ${ }^{17-19}$

One means of improving decision support is to provide more integrated access to the evidence base through clinicians' established information systems. ${ }^{2021}$ Effectiveness is increased if these systems can provide feedback via "clinical reminders" to guide the provision of care to individual patients. ${ }^{22}$ Systems can also monitor treatments and health outcomes prospectively in local or practice populations against local and national targets. ${ }^{20}$ If different local clinical systems can be linked, rapid communication of referrals and investigative results between providers and to patients is also possible. ${ }^{20}$

Despite these potential benefits and indications that electronic records are more understandable, legible, memorable and complete, ${ }^{23}$ paper based record systems remain dominant in acute care settings. ${ }^{24}$ The reluctance to move to electronic records appears increasingly untenable in the context of increasing uses and demands for patient data, more affordable and reliable computer technology, and an increased need for transferable records. ${ }^{24}$ As leading US health care reformer Don Berwick recently commented, the continued lack of systemic measurement of health care outcomes, wasteful duplication of data collection, and lack of modernised information management structures are some of the main challenges facing modern health systems. ${ }^{25}$

\section{BENEFITS OF CHD DISEASE REGISTERS IN MODERN HEALTH SYSTEMS}

Disease registers (or as they are alternatively termed, registries) offer considerable potential to facilitate the development of integrated clinical systems to support and evaluate patient care. Registers hold data that are systematically updated on people within geographically circumscribed populations with a common characteristic. ${ }^{26}$ While diabetes registers are common and evidence from the USA indicates that registers can facilitate the successful management of chronic diseases, ${ }^{20}$ the contribution that disease registers can make to $\mathrm{CHD}$ prevention and management has received comparatively little attention. ${ }^{26}$

CHD registers contain data about individuals with or at risk of CHD and should contain fields on demographics, relevant medical history, risk factor profiles, current treatments, test results, referrals, and service usage. In some settings, at least some of these data are likely to be collected and stored electronically on a routine basis through established clinical information systems. However, data on the same patient is likely to be stored in different locations that cannot readily exchange data. Consequently, data input by health professionals is often duplicated and there is little exploitation of data beyond that which is undertaken within the setting in which the data were collected or are 
stored. Even in instances where a register of CHD patients is maintained, most registers have limited links with other systems, thereby constraining their potential benefits. ${ }^{27-30}$

By collecting, collating, and processing the various data pertaining to individuals stored on different locations and systems, CHD registers are able to provide a wide range of significant and timely benefits (table 1). CHD registers can allow practitioners to have rapid access to comprehensive and up to date information, including patient's laboratory results, current pharmacotherapies, risk factors, lifestyle changes, and service usage. During consultations, current treatments can be assessed automatically and feedback given in accordance with latest guidelines and local protocols.

Throughout Europe and North America, CHD management and prevention is increasingly carried out by multidisciplinary health care teams. ${ }^{20}{ }^{31}$ Yet, care over the duration of the patient's journey is often characterised by poor communication and a lack of coordination between providers. ${ }^{32}$ Few clinical information systems currently disseminate data to other professionals working with the same patient at a different site or collate data collected by these different professionals centrally. Through wireless or net-based networks (such as NHSNet), disease registers can transfer latest data between health care professionals automatically and in real time. This allows health professionals from different disciplines in either clinical or home settings to access comprehensive and up-to-date patient information and results. This offers a means to better implement chronic disease management programmes that cross care settings, traditional roles, and sectors. ${ }^{20}{ }^{33}$

Disease registers also allow rapid analysis of population based data that, in conjunction with relevant guidelines, can identify in real time areas of suboptimal management at regional or practice levels. The ability to analyse practice specific data quickly and efficiently ${ }^{34}$ is useful in generating data for performance evaluation ${ }^{28}$ or as part of reimbursement procedures such as is required by the new General Practitioner General Medical Services Contract in the UK. ${ }^{17}{ }^{24}$

\begin{tabular}{|c|c|}
\hline Use of CHD register & Potential benefits \\
\hline Clinical consultations & $\begin{array}{l}\text { Identification of patients for review } \\
\text { Breakdown of current pharmaco-management } \\
\text { Detailed and up-to-date profiles of risk factors and } \\
\text { lifestyle patterns and changes } \\
\text { Feedback from guidelines on recommended } \\
\text { interventions and services } \\
\text { Rapid provision of laboratory test results } \\
\text { Aids provision of stage specific health promotion } \\
\text { Integrated links to guidelines and care protocols } \\
\text { Improved communication between sectors and } \\
\text { health professional groups } \\
\text { Scheduling of referral appointments at linked } \\
\text { external sites } \\
\text { Promotion of access to under-used services }\end{array}$ \\
\hline Service operations & $\begin{array}{l}\text { Generation of intelligence to inform local } \\
\text { commissioning decisions } \\
\text { Targeting of services and resources to vulnerable } \\
\text { groups and localities }\end{array}$ \\
\hline $\begin{array}{l}\text { Epidemiology and } \\
\text { audit }\end{array}$ & $\begin{array}{l}\text { Real time monitoring and comparisons of: } \\
\text { treatments and services } \\
\text { CHD } \\
\text { - high risk groups or cohorts } \\
\text { - local disease clusters and burden of CHD } \\
\text { impact of treatment and service provision } \\
\text { against evidence and local targets }\end{array}$ \\
\hline
\end{tabular}

Disease registers have the potential to allow individual practices and regions to assess the pharmacoeconomics of introducing new therapies or treatment regimens to various patient groups. The projected costs of alternative treatment at different thresholds could then be calculated and compared with established protocols. ${ }^{26}$

Cardiac services are less likely to be offered to and accessed by those in most need, such as people from deprived communities, ${ }^{35}{ }^{36}$ women ${ }^{37}$ and the elderly ${ }^{38}$ For public health and ethical reasons these disparities should be reduced. ${ }^{39}$ Disease registers can identify local trends in the availability and access to interventions and services in almost real time. Knowledge of these trends can be used to guide local commissioning decisions and target services to vulnerable or underserved populations, thereby reducing health inequalities.

\section{THE "HAVE A HEART PAISLEY" CHD REGISTER}

We created an integrated regional CHD register as part of the Scottish Executive national demonstration project for CHD "Have a Heart Paisley" (HaHP). This computerised database has been established in Paisley, Scotland (population $85000)$. It can prospectively store, receive, and send information from a variety of national and local databases used within the region hosted both in primary and secondary care settings (fig 1). The CHD register uses data warehouse technology provided by CareNet-software developed by the Centre for Health Informatics in the University of Wales ${ }^{40}$ that uses standard internet technologies and common platforms such as Microsoft NT, SQL Server, and Internet Explorer. Data transfer is achieved utilising existing interfaces built into feeder applications or, in the case of general practitioner systems, by adding a module (Clinical Decision Software System developed by Merck Sharp and Dohme), which allows the CHD register to "sit behind" the users' existing clinical information systems and supports the creation of shared care templates and data inter-interchange procedures. ${ }^{41}$

Data on each individual with CHD in the area is stored securely and ethically ${ }^{42}{ }^{43}$ in over 100 fields in categories including demographics, disease status, risk factor profile, laboratory results, pharmacological treatment, behavioural change, and service usage. These fields and their definitions were developed in consultation with the local health professionals; such involvement is integral to the design of effective clinical systems. ${ }^{24}$

Designated data fields from the CHD register can be accessed by health professionals in the region who provide care for CHD patients. The CHD register can present these professionals with the latest and relevant information on the patient obtained from different local and national sources. The specific information that different professional groups can access or amend is dependent on their needs and roles. These data can be accessed in patients' homes via the use of dedicated mini personal computers that can be linked to the CHD register.

The CHD register also offers a means to evaluate practice in a number of dimensions. Data on management and secondary prevention, including issues discussed with patients, can be extracted in real time. Practices can then monitor and compare their own patient populations, treatments, and services to regional norms and targets.

\section{CHALLENGES IN THE DEVELOPMENT OF DISEASE REGISTERS}

One of the main challenges in developing registers in health care settings is to ensure that data are processed legally and ethically. ${ }^{24}$ Disease registers must comply with the latest legislation relating to the processing of personal data, most 


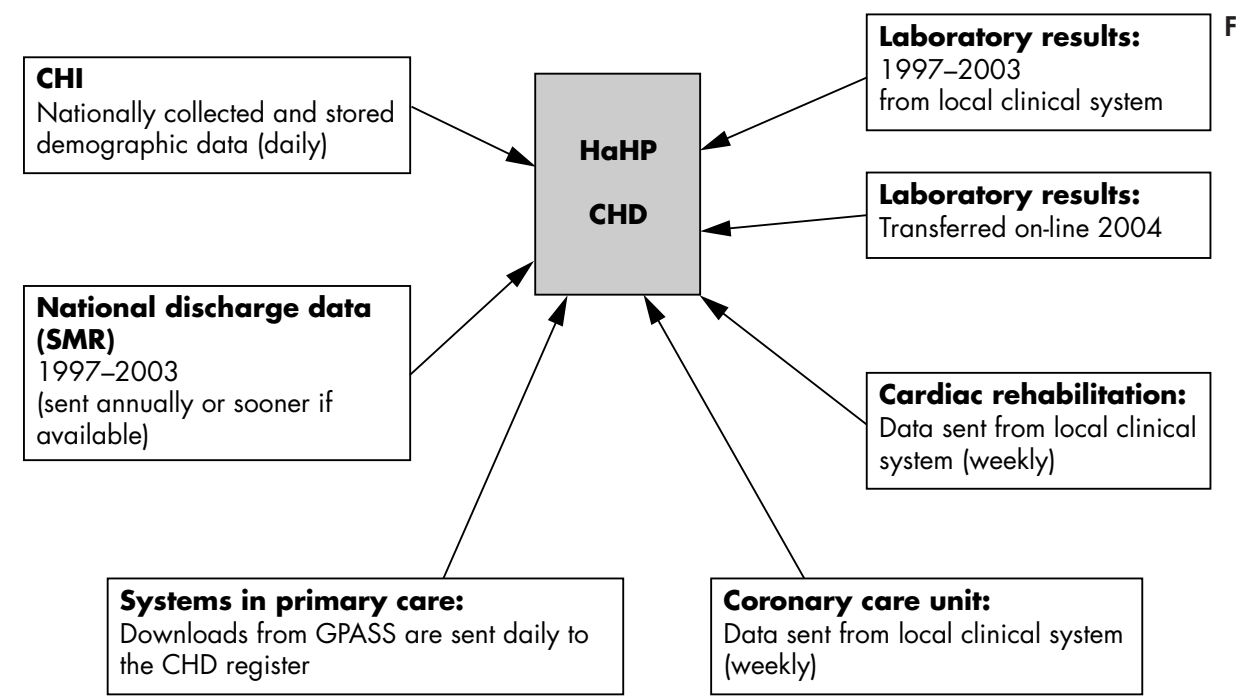

particularly the Data Protection Act (1998). In our case, no legal precedents existed to guide how the CHD register was implemented to comply with this Act. Discussions were held with a number of relevant authorities including the General Medical Council, Data Protection Act officials, and the Scottish Executive. If full written consent for inclusion were to be attained, each patient in the region would have to give written consent for the processing of personal data. The anticipated response rate from such a strategy was likely to be so low that the register could not fulfil some of its public health functions. As such, the individual's right to full consent had to be balanced with the collective benefits likely to accrue from a well populated register.

Based on advice from key agencies, a strategy was developed that focused on opting out of inclusion. A widespread publicity strategy was implemented that took all reasonable steps to inform members of the Paisley population about the CHD register, the personal data that may be stored on it, and the possible uses of these data. ${ }^{42}{ }^{43}$ Material was distributed to all households in the region, and additional leaflets and posters were placed in general practitioner surgeries, outpatient clinics, libraries, pharmacies, community centres, and local benefits offices. Individuals who did not wish to be included on the CHD register could opt out via a local telephone number or by using a freepost address.

\section{CONCLUSION}

Greater support is needed to facilitate evidence based and equitable cardiac care.

Effective utilisation of computerised information technologies is vital to achieving these ends but is poorly done in many instances. CHD registers offer considerable potential to provide increased support to practitioners, facilitate improvements in patient care, and allow real time and efficient systemic monitoring of care provision and outcomes. These benefits accrue at different levels and address directly a range of significant, long established, and notoriously intractable problems. The HaHP CHD register demonstrates that it is feasible to develop a well linked CHD register that uses common applications to address these challenges.

\section{ACKNOWLEDGEMENTS}

The HaHP CHD register is funded through the Scottish Executive national demonstration project, "Have a Heart Paisley". The views expressed are those of the authors.

\section{Authors' affiliations}

A M Clark, University of Alberta, Edmonton, Alberta, Canada I N Findlay, Royal Alexandra Hospital, Paisley, UK

Conflict of interest: None

\section{REFERENCES}

1 Gray J. Evidence-based healthcare. London: Churchill, Livingstone, 1997.

2 Komaida M, Follath F, Swedberg K, et al. The EuroHeart Failure Survey programme-a survey on the quality of care among patients with heart failure in Europe. Part 2: treatment. Eur Heart J 2003;24:464-74.

3 Euroaspire II Study Group. Lifestyle and risk factor management and use of drug therapies in coronary patients from 15 countries. Eur Heart $J$ 2001;22:554-72.

4 Hippisley-Cox J, Pringle M. Inequalities in access to coronary angiography and revascularisation: the association of deprivation and location of primary care services. Br J General Pract 2000;50:449-54.

5 Pell JP, Pell ACH, Norrie J, et al. Effect of socioeconomic deprivation on waiting time for cardiac surgery: retrospective cohort study. Commentary: three decades of the inverse care law. BMJ 2000;320:15-19.

6 MacLeod MCN, Finlayson AR, Pell JP, et al. Geographic, demographic, and socioeconomic variations in the investigation and management of coronary heart disease in Scotland. Heart 1999;81:252-6.

7 Department of Health. National service framework for coronary heart disease. London: Department of Health, 2000

8 SIGN. Guideline 35: Diagnosis and treatment of heart failure due to left ventricular systolic dysfunction. Edinburgh: SIGN, 1999.

9 SIGN Guidelines Network. Secondary prevention of coronary heart disease. Edinburgh: SIGN Secretariat, 2000.

10 Clinical Standards Board for Scotland. Secondary prevention following myocardial infarction. Edinburgh: Clinical Standards Board for Scotland 2000.

11 Anon. Third joint task force of European and other societies on cardiovascular disease prevention in clinical practice. European guidelines on cardiovascular disease prevention in clinical practice. Eur Heart J 2003;24:1601-10.

12 Mosca L, Appel L, Benjamin EJ, et al. Evidence-based guidelines for cardiovascular disease prevention in women. Circulation 2004;109:672-93.

13 Bodenheimer T, Grumbach K. Electronic technology - a spark to revitalise primary care? JAMA 2003;290:259-64.

14 Hobbs FR, Erhardt L. Acceptance of guideline recommendations and perceived implementation of coronary heart disease prevention among primary care physicians in five European countries: the reassessing European attitudes about cardiovascular treatment (REACT) survey. Family Practice 2002;19:596-604

15 Majumdar SR, McAlister FA, Furberg CD. From knowledge to practice in chronic cardiovascular disease: a long and winding road. J Am Coll Cardiol 2004;43:1738-10.

16 Delaney BC, Fitzmaurice DA, Riaz A, et al. Can computerised decision support systems deliver improved quality in primary care? BMJ 1999;319:1281.

17 NHS Confederation. Investing in general practice: the new general medicine services contract. London: NHS Confederation, 2003.

18 Department of Health. The NHS plan. HMSO: London, 2000

19 British Cardiac Society. Fifth report on the provision of services for patients with heart disease. Heart 2002;88(suppl III):iiil-59.

20 Bodenheimer T, Wagner EH, Grumbach K. Improving primary care for patients with chronic illness. JAMA 2002;288:1775-9.

21 Department of Health. Draft national patient information charter. London: Department of Health, 2002 
22 Hunt DL, Haynes RB, Hanna SE, et al. Effects of computer-based clinical decision support systems on physician performance and patient outcomes: systematic review. JAMA 1998;280:1339-46.

23 Hippisley-Cox J, Pringle M, Cater R, et al. The electronic patient record in primary care-regression or progression? A cross sectional study. BMJ 2003;326: 1439-43.

24 National Academy of Sciences. The computer-based patient record: an essential technology for health care. London: National Academy of Sciences, 2003.

25 Vastag B. Donald M. Berwick, MD, MPP: advocate for evidence-based health system reform, JAMA 2003;291:1945-7.

26 Newton J, Garner S. Disease registers in England. Oxford: Institute of Health Sciences, 2002.

27 Moher M, Yudkin P, Turner R, Schofield T, for the ASSIST collaborative group. An assessment of morbidity registers for coronary heart disease in primary care. J Clin Nursing 2000;315:69-70.

28 Carroll K, Majeed A, Firth C, et al. Prevalence and management of coronary disease in primary care: population-based cross-sectional study using a disease register. Br J General Practice 2003;25:29-35.

29 Horsfield $\mathbf{P}$. Making the most of your disease registers is key to quality points. Pulse 2003;69(41):2-3.

30 Begg AG, Grittith JM. The electonic health record and the management of cardiovascular disease. Br J Cardiol 2002;9:630-3.

31 Grady KL, Dracup K, Kennedy G, et al. Team management of patients with heart failure. Circulation 2000;102:2443-56.

32 Bethell HJ, Turner SC, Evans JA, et al. Cardiac rehabilitation in the United Kingdom. How complete is the provision? Journal of Cardiopulmonary Rehabilitation 2001;21:111-5.
33 Scottish Executive. Partnership for care. Edinburgh: Scottish Executive, 2003

34 Gray J, Ekins M, Scammell S, et al. Workload implications of identifying patients with ischaemic heart disease in primary care: population-based study. J Public Health Med 2003;25:223-9.

35 Melville MR, Packham C, Brown C, et al. Cardiac rehabilitation: socially deprived patients are less likely to attend but patients ineligible for thrombolysis are less likely to be invited. Heart 1999;82:373-7.

36 McAlister FA, Murphy NF, Simpson CR, et al. Influence of socioeconomic deprivation on the primary care burden and treatment of patients with a diagnosis of heart failure in general practice in Scotland: population based study. BMJ 2004;328:1110.

37 Cooper AF, Jackson G, Weinman J, et al. Factors associated with cardiac rehabilitation attendance: a systematic review of the literature. Clinical Rehabilitation 2002;16:541-52.

38 Clark AM, Sharp C, Maclntyre PD. The role of age in moderating access to cardiac rehabilitation in Scotland. Ageing and Society 2002;22:501-15.

39 Department of Health. National service framework for older people. London: Department of Health, 2001

40 Centre for Health Informatics. http://www.chi.swan.ac.uk.

41 Anon. Product description of Have a Heart Paisley Central data Repository http://www. show.scot.nhs.uk/hahpregister/HaHP.

42 Clark AM, Blatchford O, Jamieson R, et al for the HaHP IT Group. Ensuring the ethics and legality of disease registers: the solution of the Have a Heart Paisley Register. British Journal of Health Care Computing \& Information Management 2003;20(2):44-8

43 Clark AM, Jamieson R, Findlay I. Registries and informed consent. N Engl J Med 2004;351:612.

\section{IMAGES IN CARDIOLOGY}

\section{A case of anomalous origin of the right coronary artery from the pulmonary artery complicated by acute myocardial infarction}

A

77 year old man was brought to our hospital with an episode of dyspnoea on 29 December 2003, and hospitalised on the following day. The chest radiograph indicated cardiomegaly and pulmonary oedema, and the ECG showed a QS pattern and an elevated ST segment in V1-V3 and a depressed ST segment in V5 and V6. Although he was diagnosed as having congestive heart failure caused by acute myocardial infarction, we did not perform emergency cardiac catheterisation because too much time had passed from the onset of the disease. There was a mild elevation in max creatine kinase (CK) $(521 \mathrm{iu} / \mathrm{l})$ and in max CK-MB (61 iu/l). We performed diagnostic cardiac catheterisation on 26 January 2004. Left ventriculography indicated that contraction was absent in segments 2, 3, 4, and 6 and that the ejection fraction was $26 \%$. Left coronary angiography indicated $100 \%$ stenosis in number $7,90 \%$ in number 13 , $75 \%$ in number 14 , and $90 \%$ in number 15 . Since there was a chronic complete occlusion in number 7 , the left circumflex branch was most likely involved in the infarction. Some of the blood in the left circumflex branch and the left anterior descending branch was flowing into the pulmonary artery via the right coronary artery. We performed contrast enhancement multidetector row computed tomography (MDCT) of the chest on 27 January 2004. We obtained detailed images that clearly indicated that the right coronary artery had an anomalous origin from the pulmonary artery (panel: Ao, aorta; LAD, left anterior descending coronary artery; PA, pulmonary artery; RCA, right coronary artery). Since the left anterior descending branch was completely occluded and the left circumflex branch was severely stenotic, coronary artery bypass graft surgery (CABG) was judged to be appropriate, and the patient was transferred to the referral hospital. He underwent CABG for two branches (LITA-ltRA-PL1-PL2) and left ventriculoplasty. We gave up on bypass graft surgery for

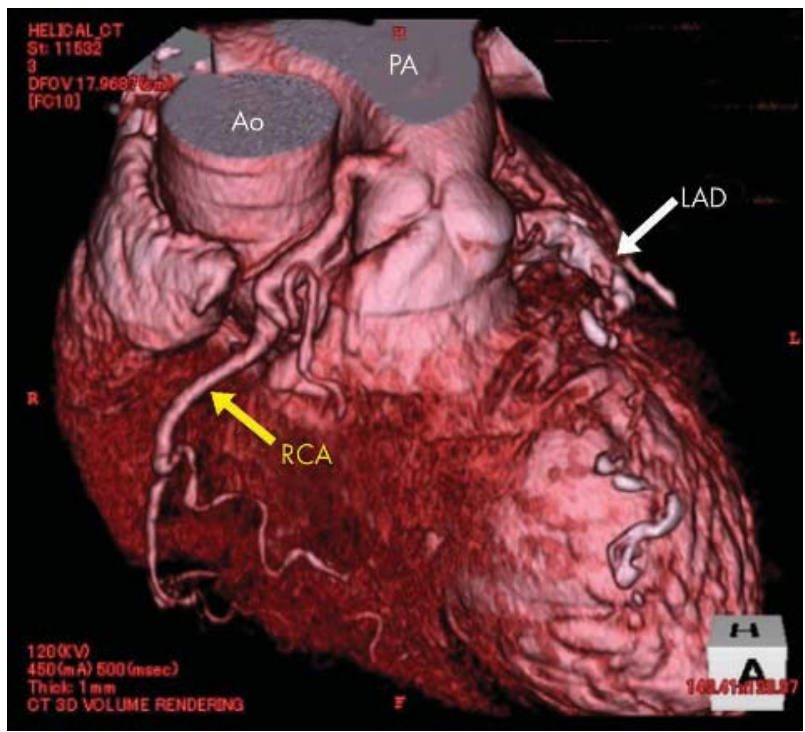

the left anterior descending branch because of the remarkable calcification and stenosis present. We decided to follow up the course of the right coronary artery disease with palliative treatment only. 